\title{
Online Acquisition and Visualization of Motion Primitives for Humanoid Robots
}

\author{
Dana Kulić, Hirotaka Imagawa and Yoshihiko Nakamura
}

\begin{abstract}
This paper proposes an on-line, interactive approach for incremental learning and visualization of full body motion primitives from observation of human motion. The human demonstrator motion is captured in a motion capture studio. The continuous observation sequence is first partitioned into motion segments, using stochastic segmentation. Motion segments are next incrementally clustered and organized into a hierarchical tree structure representing the known motion primitives. At the same time, the sequential relationship between motion primitives is learned, to enable the generation of coherent sequences of motion primitives. An on-line visualization system is also developed to allow the demonstrator to visualize the motion database and the motion primitives learned by the system, thus giving the demonstrator insight into the learning process and the ability to interactively modify the demonstration based on the current state of the knowledge base. The developed system has many potential applications for motion analysis, prediction and imitation learning for humanoid robots.
\end{abstract}

\section{INTRODUCTION}

The ability to extract knowledge about human motion and motion primitives through continuous observation of human behavior is an attractive paradigm, with many potential applications. For humanoid robots, such a system would enable the robot to learn how to accomplish tasks by simply observing a human demonstrator, and therefore take advantage of the similarity in body structure between humanoids and humans, avoiding the need for explicit programming of complex robot motions. In order to extract motion primitives and behaviors during on-line observation, several key issues must be addressed by the learning system: automated motion segmentation, recognition of previously learned motion primitives, automatic clustering and learning of new motion primitives, and finally, learning how motion primitives can be combined into sequences to form behaviors. We have been developing an approach for on-line segmentation and clustering of whole body human motion primitives, and incremental learning of the relationship between the motion primitives for the formation of longer behaviors [1] [2] [3]. The observed motion time series data stream is first stochastically segmented into potential motion primitive segments, based on the assumption that data belonging to the same motion primitive will have the same underlying distribution.

Dana Kulić was with the Department of Mechano-Informatics, University of Tokyo, and is now with the Department of Electrical and Computer Engineering, University of Waterloo, 200 University Avenue West, Waterloo, Ontario N2L 3G1, Canada. Hirotaka Imagawa and Yoshihiko Nakamura are with the Department of Mechano-Informatics, University of Tokyo, 7-3-1 Hongo, Bunkyoku, 113-8656 Tokyo, Japan dkulic@ece.uwaterloo.ca, \{imagawa, nakamura\}@ynl.t.u-tokyo.ac.jp
The segmented motions are then passed to an incremental clustering algorithm which forms a tree representation of the learned motions, and abstracts each motion type into a generative model. Concurrently, a graph model is built representing the sequential relationship between the motion primitives. The graph can then be used to generate new coherent motion sequences for the robot, based on the learned motion primitives and the learned relationship between them. In addition to the application to robot motion generation, the developed automated motion primitive system can also be utilized for motion analysis during sports training or rehabilitation, activity detection as well as human motion behavior understanding and prediction.

In addition to its capabilities for incremental learning and motion abstraction, to ensure ease of use during training by a human teacher, the system must have some mechanism for representing and displaying the knowledge acquired so far. In this paper we propose a system for visualizing the motion primitive database and motion primitive graph during acquisition. The proposed system allows the human demonstrator or user to have a better insight into the current status of the robot's knowledge. The improved understanding of the learning system and learning progress facilitates the training process for the human demonstrator, by allowing the demonstrator to easily grasp what the system has learned so far and how that knowledge has been organized. The demonstrator can use the visualized information to guide further training, such as providing additional or corrective examples, or modifying the training sequence to better elucidate the sequential connections between motion primitives.

\section{A. Related Work}

Breazeal and Scasellati [4] and Schaal et al. [5] provide reviews on motion learning by imitation. As noted by Breazeal and Scasellati, the majority of algorithms discussed in the literature assume that the motions to be learned are segmented and clustered a-priori, and that the model training takes place off-line. Since the material to be learned is defined a-priori and analyzed off line, there is little need for online visualization of the learning process.

Nakaoka et al. [6] develop an approach for learning and executing dance motion sequences for a humanoid robot. They also develop a visualization system for visualizing the behavior in simulation, prior to re-targeting the motions on a humanoid robot. However, all the exemplar motion patterns are acquired and grouped before the training begins, and the number of motions to be learned is specified a priori. 
Taylor et al. [7] describe an approach for modeling human motion using a conditional restricted Boltzmann machine (CRBM). The learned model can generate continuous motion sequences, as well as learn the transitions between motions. Once the low level model consisting of individual motion patterns has been trained, additional higher order layers can be added to model the higher order structure of motion patterns. However, this implies that all primitive level motions must be known and input into the model, so that the algorithm is not able to build the model incrementally, during on-line observation.

Jenkins and Matarić [8] describe a system for extracting behaviors from motion capture data. In their algorithm, continuous time series data is first segmented using the kinematic centroid segmentation algorithm. The segmented data is then embedded in a lower dimensional space using the spatio-temporal Isomap algorithm [9]. The lower dimensional space can also be used to visualize the motion data. Once the data has been reduced, it is clustered into groupings using the "sweep-and-prune" technique. While this system autonomously segments and clusters data, the algorithm cannot operate incrementally, as the entire range of motions is required to form the lower-dimensional space embedding.

Hidden Markov Models have been a popular technique for human motion modeling, and have been used in a variety of applications, including skill transfer [10], sign language and gesture modeling [11] and motion representation [10] [12]. A common paradigm is Programming by Demonstration (PbD) [10]. In previous research [13], [14], [15], [16], humanoid motion primitives have been encoded using Hidden Markov Models, and subsequently used for motion generation. However, the initial training of the models was carried out offline, where all the training examples for each model were grouped manually.

Calinon et al. [12] describe a system for programming by demonstration based on Gaussian Mixture Models. This system is also extended to an on-line, interactive approach by developing a method for incremental training of the GMM structure [17] and developing an interactive training approach combining demonstration and kinesthetic training [18]. However it appears that the motion segmentation is performed manually by the trainer.

Outside the robotics community, in the computer graphics domain, there has also been a long standing research effort to develop algorithms for realistic human-line motion generation for animated characters. Kovar et al. [19] first proposed the motion graph technique. In this approach, a directed graph is constructed encapsulating the relationships between postures extracted from a motion capture data set. The graph can then be used to generate extended sequences of realistic looking motions. Yamaguchi et al. [20] develop an algorithm for building a motion graph via a binary tree clustering technique.

\section{B. Proposed Approach}

The aim of our research is to develop robots which can learn motion primitives and higher level behaviors on-line while observing and interacting with a human partner over extended periods of time. Using continuous time-series data as the input, we first segment the data into potential motion primitives, using a modified version of the Kohlmorgen and Lemm [21] algorithm for unsupervised segmentation. Next, the extracted segments are input into an automated clustering and hierarchical organization algorithm [22] [23] [1]. Individual motion patterns are clustered in an incremental fashion, based on intra model distances. Concurrently with the learning of the motion primitives, the relationship between primitives is learned by forming a directed graph of the motion primitives [3]. The motion primitive graph can then be used for subsequent motion sequence generation.

During the acquisition of the motion primitives database, the demonstrator is able to visualize the state of the knowledge database through the visualization system. This system provides a visual overview of the motion database, the motion primitive graph, and individual motion primitives. Section 2 summarizes the segmentation, clustering and motion primitive graph formation algorithms, while Section 3 describes the on-line visualization system. In Section 4, the results of experiments verifying the algorithm on a continuous stream of human motion capture data is reported. Section 5 concludes the paper and provides directions for future work.

\section{ON LINE SEGMENTATION, CLUSTERING AND MOTION PRIMITIVE GRAPH FORMATION}

In the proposed approach [1], [2], [3], the on-line learning system autonomously segments, clusters and learns the sequencing of full-body motion primitives from on-line observation of full body human motions.

\section{A. Segmentation}

First, the incoming continuous time series data is segmented into potential motion primitive segments. The Kohlmorgen and Lemm segmentation algorithm [21], [24] is based on the assumption that data belonging to the same motion primitive will have the same underlying probability distribution. The incoming data stream is first embedded into a higher-dimensional space. Next, the density distribution of the embedded data is estimated over a sliding window of length $W$, via a standard density estimator with multivariate Gaussian kernels. As more data are observed, the distance between successive data windows can be calculated based on the integrated square error between two probability density functions:

$$
d\left(p_{t 1}, p_{t 2}\right)=\int\left(p_{t 1}(x)-p_{t 2}(x)\right)^{2} d x
$$

This distance can be calculated analytically in the case of mixtures of Gaussian density functions. The segmentation points can then be estimated by defining a Hidden Markov Model over a set $S$ of sliding windows. Each window 
corresponds to a state of the HMM. For each state, the observation probability distribution is defined as:

$$
p\left(p_{t}(\mathbf{x}) \mid s\right)=\frac{1}{\sqrt{2 \pi} \varsigma} \exp \left(-\frac{d\left(p_{s}(\mathbf{x}), p_{t}(\mathbf{x})\right)}{2 \varsigma^{2}}\right),
$$

where $p\left(p_{t}(\mathbf{x}) \mid s\right)$ is the probability of observing the window represented by $p_{t}(\mathbf{x})$ in state $s$. The initial state distribution is given by the uniform distribution, and the state transition matrix is designed such that transitions to the same state are $k$ times more likely than transitions to any of the other states.

$$
a_{i j}= \begin{cases}\frac{k}{k+N-1} & \text { if } i=j \\ \frac{1}{k+N-1} & \text { if } i \neq j .\end{cases}
$$

where $N$ is the number of states of the HMM. The Viterbi algorithm [25] can then be used to find the optimum state sequence given the current set of observations. An on-line variant of the Viterbi algorithm is also developed [21], which incrementally builds the state path table as each new state is observed, by re-using the the estimate of the likelihood and optimal state sequence from the previous time step.

\section{B. Incremental Clustering}

Once the incoming time series data has been segmented into potential primitives, each segment is sequentially passed to the clustering module. In the proposed clustering approach [22], [23], a hierarchical tree structure is incrementally formed representing the motions learned by the robot. Each node in the tree represents a motion primitive, which can be used to recognize a similar motion, and also to generate the corresponding motion for the robot. Within each local area of the motion space, a standard clustering technique [26] is used to subdivide motion primitives. A Hidden Markov Model is used to abstract the observation sequences. The parameters of the model form the feature set of the data. These features are then used to define a distance measure between observation sequences, which is used for clustering.

The algorithm initially begins with one group (the root node). Each time a motion is observed from the teacher, it is encoded into an HMM and compared to existing groups via a tree search algorithm, and placed into the closest group. Each time a group is modified, local clustering is performed within the exemplars of the group. If a a cluster with sufficiently similar data is found, a child group is formed with this data subset. Therefore the algorithm incrementally learns and organizes the motion primitive space, based on the robot's lifetime observations. The algorithm is overviewed in Fig. 1.

This algorithm allows the robot to incrementally learn and classify motion primitives observed during continuous observation of a human demonstrator. The robot's knowledge is organized based on the type of training received, so that the robot's knowledge will be most specialized in those areas of the motion primitive space where the most data has been observed.

Once a cluster node has been formed, the group model for the node constitutes the abstraction of the motion primitive. To generate a motion trajectory for the robot from the group model, the deterministic motion generation method is used [27]. In this method, at each time step, the state duration is first estimated from the state transition model, and the subsequent state is selected by a greedy policy. The output observation vector is then generated by a greedy policy on the output model. The resulting reference trajectory is then low-pass filtered and passed to a low level controller, to ensure that dynamic and stability constraints are satisfied.

\section{Motion Primitive Graph Formation}

Concurrently with the construction of the hierarchical tree structure representing the motion primitives, we learn the relationship between the primitives by constructing a directed graph representing the observed transitions between the primitives. Each node in the motion primitive graph represents a motion primitive, while each edge represents an observed transition between two motion primitives.

Initially, the graph is empty, as no motion primitives are known at initialization. Each time a new motion primitive is abstracted by the clustering algorithm as a leaf node (described in Section III), a corresponding node is added to the motion primitive graph. The incremental clustering algorithm also performs motion recognition. When a newly observed motion segment is placed in an existing (non-root) node of the tree, this indicates that the motion segment has been recognized as the motion primitive corresponding to the selected node. A motion primitive transition model is built incrementally by monitoring for instances when a sequence of two motion primitives are recognized by the incremental clustering algorithm. Each time a recognized motion primitive transition is detected, the corresponding edge is incremented. In this way, the robot incrementally learns how motion primitives may be combined during behavior execution.

The constructed graph can then be used to generate sequences of primitives by concatenating a set of nodes connected in the graph, for example, by searching the graph for a valid path given a starting and target position. The graph can also be used to generate novel sequences of primitives, not observed from the demonstrator. In this way, the robot can generate novel behaviors based on its known motion primitives and their relationships.

\section{VisUALIZATION}

During the demonstration process, (as well as following learning), it is important for the teacher to have visibility into the material learned so far, so that the teacher can tailor further demonstrations to the current knowledge of the learner. The importance of user insight into the autonomous system operation has already been extensively investigated in the knowledge and data discovery community (KDD) [28]. This research has found that the visualization of expert system knowledge in an easily comprehensible form is a key requirement for system useability and acceptance by human users. In addition, in the KDD domain, the visualization of expert system output allows the user to not only understand 


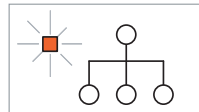

(a)

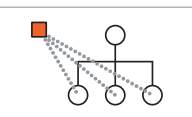

(b)

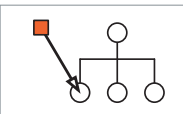

(c)

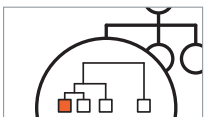

(d)

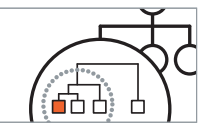

(e)

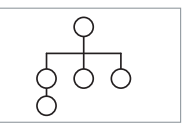

(f)

Fig. 1. Overview of the Incremental Clustering Algorithm (A square represents a data sequence, and a circle represents a group). (a) a new observation sequence is observed and encoded as an HMM; (b) the observation sequence is compared to existing groups via tree search; (c) the new sequence is placed in the closest existing group; (d) local clustering is performed on the modified group (zoomed in view of modified group); (e) a new subgroup is formed from similar motions in the modified group; (f) the subgroup is added to the tree as a child of the modified group.

the expert system analysis, but also to combine the autonomous analysis with human analysis to achieve additional insight. Similarly to the KDD domain, in the proposed robot programming by demonstration approach, an autonomous system analyzes a large corpus of demonstrator data and extracts data abstractions in the form of motion primitives and their sequencing. In order to enhance useability and user acceptance of the system, both the process and the resulting data structure should be made clearly visible to the human user. Moreover, understanding the current knowledge of the system allows the demonstrator to combine the insight of the autonomous system with his or her own knowledge to improve the training process and the resulting knowledge base obtained by the robot learner. In this paper, we report on the development of a visualization system capable of presenting the learned data to the user on-line, during demonstration. To the authors' knowledge, this is the first such system for visually presenting the robot's acquired knowledge to the demonstrator during on-line interaction. Note that the visualization system is enabled by the capability of the learning algorithm described in Section II to operate in realtime. In the developed system, at any time during the demonstration, as well as off-line, the human demonstrator is able to view animations of the motion primitives acquired thus far, an overview of the current tree structure of the database, and a visualization of the current version of the motion primitive graph. During the acquisition process itself, the demonstrator views an animation of the motion currently being executed, and is also notified when the system has recognized the current motion primitive as one of the known motions.

The visualization system receives the marker data from the motion capture system directly. The marker data is next converted to joint angle data via on-line inverse kinematics [29], based on the kinematic model of the animation character. The animation character, in terms of the body shape and the number of degrees of freedom, can be selected based on the requirements of the task being demonstrated in the case of motions being acquired for human motion analysis, or based on the kinematic structure of the robot, in the case of motions being acquired for later re-targeting to the humanoid robot. In the implementation described here, we select a $43 \mathrm{DoF}$ model. This model was selected because it has been found to provide a good tradeoff between model complexity and the ability to adequately represent most daily activities [30].

The current motion of the demonstrator is displayed on the animated character simultaneously with motion execution.
This function is useful to allow the demonstrator to verify that the inverse kinematics is working properly and that motions are being re-targeted to the humanoid joint angle data correctly, without inducing representational singularities. Figure 2 shows a screen shot of the visualization system during motion acquisition.

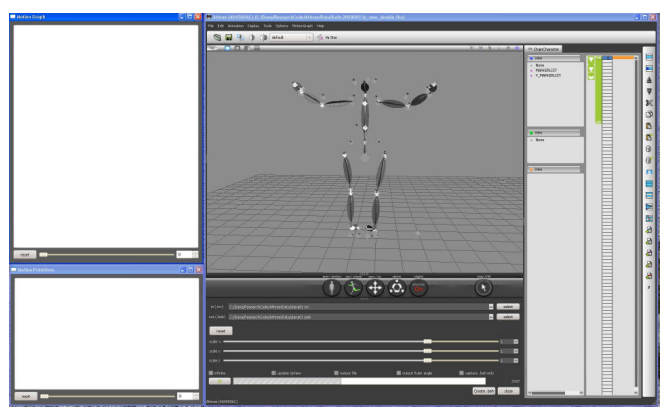

Fig. 2. Screen shot of the visualization software during motion acquisition

The joint angle data is then passed to the segmentation, clustering and motion primitive graph learning algorithm, as described in Section II. Each time a new motion primitive is abstracted, a node is added to the tree structure representing the current status of the knowledge database, as well as the motion primitive graph. The tree structure and motion primitive graph can be viewed at any time within the visualization system; the formation of the first node during training is shown in Figure 3.

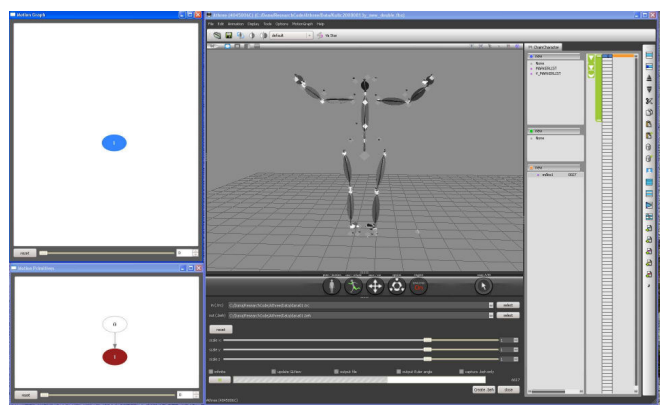

Fig. 3. Screen shot of the visualization software during node formation

Each time a previously learned motion primitive is recognized in the current demonstration, the visualization system notifies the demonstrator that the performed motion is already known to the system. This is indicated (see Figure 4) by changing the color of the identified node in the tree and motion primitive graph. If two known motions are recognized in succession, the motion primitive graph is updated. A 
motion primitive graph update is shown in Figure 5. The GraphViz software [31] is used to perform online drawing of the directed motion primitive graph.

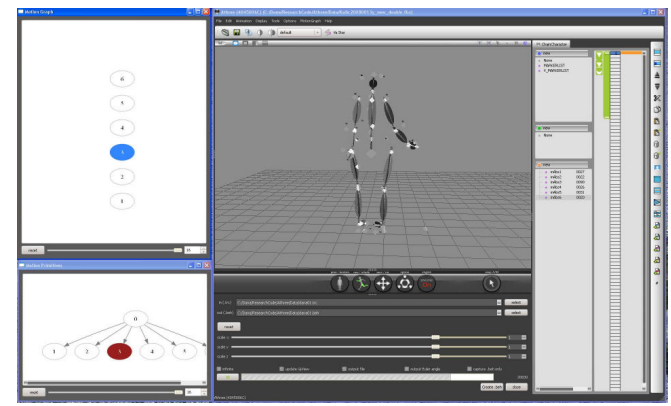

Fig. 4. Screen shot of the visualization software during motion recognition

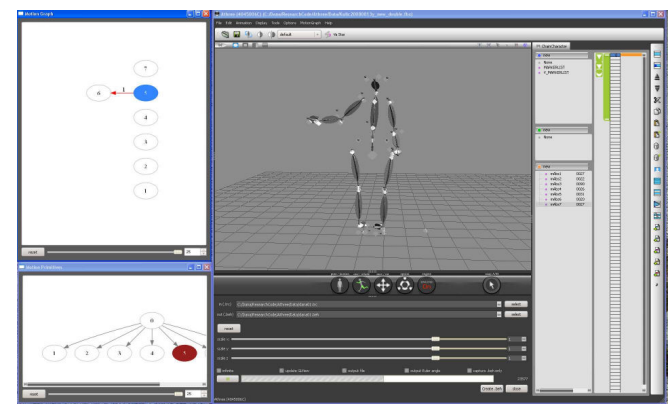

Fig. 5. Screen shot of the visualization software during motion primitive formation

Once the demonstration is over, the user can load all the learned motions (behaviors), and play them back on the animated character to verify that motion primitives have been abstracted correctly.

\section{EXPERIMENTS}

The combined segmentation, clustering and motion primitive graph extraction algorithm was tested with the visualization system using data collected in the motion capture system. To test the combined system, we collected a motion sequence with a variety of full body motions in the motion capture studio. The human demonstrator is outfitted with 34 reflective markers located on various parts of the body, and the marker $[\mathrm{x}, \mathrm{y}, \mathrm{z}]$ position is captured and computed by the motion capture system online, at a sample rate of $5 \mathrm{~ms}$. The marker positioning and experimental setup are shown in Figure 6. The demonstrator performs single and both arm raise, bow and squat motions. The demonstrator performs approximately 6 repetitions of each motion type.

The marker data is then passed to the combined motion extraction and visualization system, which performs the online inverse kinematics to generate joint angle data, displays an animation character performing the demonstrator motions, and simultaneously passes the data to the segmentation, clustering and motion primitive extraction module for processing. In previous work, a simplified inverse kinematics model was used $(20 \mathrm{DoF})$, however, here we use a more realistic 40
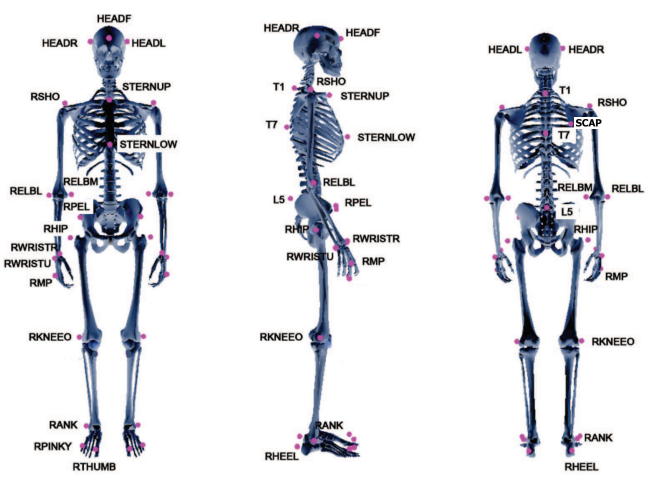

Fig. 6. Marker Setup used for the motion capture experiments

DoF model, which is better able to capture the full range of human motion. Screen shots illustrating the visualization system appearance during motion acquisitions are shown in Figures 2, 3, 4 and 5.

Following the completion of the demonstration (approximately 2.5 minutes of data), the system has learned 7 of the 10 motions demonstrated. The resulting tree structure is shown in Figure 7. The single arm motions (both the raise and the lower) are not yet differentiated, but are instead grouped together in nodes 3 and 4. Further examples of the bend raise and single arm motions result in subsequent differentiation and abstraction of these motions as well.

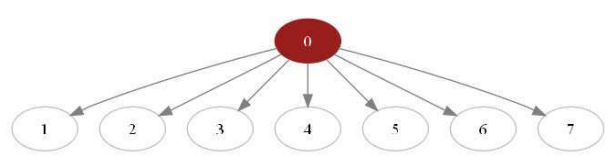

Fig. 7. Final tree structure following 2.5 minutes of data. Node 1 represents the both arms raise primitive, Node 2 represents the both arms lower primitive, Node 3 represents left arm motions, Node 4 represents right arm motions, Node 5 represents Squat Raise, Node 6 represents Squat Lower, and Node 7 represents the Bend down motion

The developed system is able to autonomously extract common motion primitives from on-line demonstration consisting of a continuous sequence of behaviors, as well as visualize the extracted motions to the demonstrator, allowing the demonstrator insight into the learning process, and the ability to interactively adjust further training.

\section{CONCLUSIONS}

This paper presents an approach for online, continuous learning of full body motion primitives and allowable motion primitive sequencing through observation of a human demonstrator, and an accompanying visualization system for assisting the user during training. The observed human motion is first converted into robot joint angle data via online inverse kinematics. The joint angle data is then autonomously segmented into potential motion primitives using stochastic segmentation [21], [32]. Segmented motion primitive candidates are incrementally clustered to abstract 
generative models of the motion primitives [2]. As each motion primitive is learned, it is also added to a motion primitive graph, which is incrementally updated to learn the relationship and sequencing rules of the motion primitives. The algorithm is capable of learning in realtime, during observation of the demonstrator's motions. This enables an interactive implementation of the learning system. To enable such an interactive learning process, a visualization system was developed giving the demonstrator insight into the current status of the learner. The visualization system allows the demonstrator to visualize the training process, by notifying the user when a new motion has been learned, when a known motion has been recognized, and when a new relationship between motions has been acquired. Following a demonstration, the visualization system also allows the user to review the material learned, and tailor future demonstrations appropriately.

In future work, the system will be validated in longer interactive sessions, with different human demonstrators, to verify the useability of the system and to analyze the variability in teaching style between different users. We will also work on developing learning methods for modeling longer sequences of primitives, which can be used to learn longer behaviors composed of motion primitives. The robot can then use the knowledge of these behaviors to predict future human motion as well as offer assistance with task execution if appropriate.

\section{ACKNOWLEDGMENTS}

The authors gratefully acknowledge the assistance of Akihiko Murai with the data set collection. This work was supported by the Japanese Society for the Promotion of Science Category S Grant-in-Aid for Scientific Research 20220001.

\section{REFERENCES}

[1] D. Kulić, W. Takano, and Y. Nakamura, "Incremental learning, clustering and hierarchy formation of whole body motion patterns using adaptive hidden markov chains," Int. Journal of Robotics Research, vol. 27, no. 7, pp. 761-784, 2008.

[2] _ - "Combining automated on-line segmentation and incremental clustering for whole body motions," in Proc. IEEE Int. Conf. on Robotics and Automation, 2008, 2591-2598.

[3] D. Kulić, D. Lee, Ch. Ott, and Y. Nakamura, "Incremental learning of full body motion primitives for humanoid robots," in Proc. IEEE Int. Conf. on Humanoid Robots, 2008, pp. 326-332.

[4] C. Breazeal and B. Scassellati, "Robots that imitate humans," Trends in Cognitive Sciences, vol. 6, no. 11, pp. 481-487, 2002.

[5] S. Schaal, A. Ijspeert, and A. Billard, "Computational approaches to motor learning by imitation," Phil. Transactions of the Royal Society of London B: Biological Sciences, vol. 358, pp. 537 - 547, 2003.

[6] S. Nakaoka, A. Nakazawa, K. Yokoi, and K. Ikeuchi, "Leg motion primitives for a humanoid robot to imitate human dances," Journal of Three Dimensional Images, vol. 18, no. 1, pp. 73-78, 2004.

[7] G. W. Taylor, G. E. Hinton, and S. Roweis, "Modeling human motion using binary latent variables," in Proc. Conf. on Neural Information Processing Systems, 2006, pp. 1345-1352.

[8] O. C. Jenkins and M. Matarić, "Performance-derived behavior vocabularies: Data-driven acquisition of skills from motion," Int. Journal of Humanoid Robotics, vol. 1, no. 2, pp. 237-288, 2004.

[9] — - "A spatio-temporal extension to isomap nonlinear dimension reduction," in Proc. Int. Conf. on Machine Learning, 2004, pp. 441448.
[10] R. Dillmann, O. Rogalla, M. Ehrenmann, R. Zollner, and M. Bordegoni, "Learning robot behaviour and skills based on human demonstration and advice: The machine learning paradigm," in Proc. Int. Symposium on Robotics Research, 1999, pp. 229-238.

[11] T. Startner and A. Pentland, "Visual recognition of american sign language using hidden markov models," in Proc. Int. Conf. on Automatic Face and Gesture Recognition, 1995, pp. 189-194.

[12] S. Calinon, F. Guenter, and A. Billard, "On learning, representing and generalizing a task in a humanoid robot," IEEE Trans. on Systems, Man and Cybernetics, Part B, vol. 37, no. 2, pp. 286-298, 2007.

[13] A. Billard, S. Calinon, and F. Guenter, "Discriminative and adaptive imitation in uni-manual and bi-manual tasks," Robotics and Autonomous Systems, vol. 54, pp. 370-384, 2006.

[14] H. Ezaki, "Understanding actions of others through self-action components," Master's thesis, University of Tokyo, 2000, in Japanese.

[15] T. Inamura, I. Toshima, H. Tanie, and Y. Nakamura, "Embodied symbol emergence based on mimesis theory," The Int. Journal of Robotics Research, vol. 23, no. 4-5, pp. 363-377, 2004.

[16] D. Lee and Y. Nakamura, "Stochastic model of imitating a new observed motion based on the acquired motion primitives," in Proc. IEEE Int. Conf. on Intelligent Robots and Systems, 2006, pp. 49945000 .

[17] S. Calinon and A. Billard, "Incremental learning of gestures by imitation in a humanoid robot," in Proc. ACM/IEEE Int. Conf. on Human-Robot Interaction, 2007, pp. 255-262.

[18] — , "Active teaching in robot programming by demonstration," in Proc. IEEE Int. Conf. on Robot and Human Interactive Communication, 2007, pp. 702-707.

[19] L. Kovar, M. Gleicher, and F. Pighin, "Motion graphs," in Proc. ACM SIGGRAPH, 2002, pp. 473-482.

[20] Y. Yamaguchi, K. Yamane, and Y. Nakamura, "A large-scale human motion database and its application to character animation," in Proc. JSME Conf. on Robotics and Mechatronics, 2008, pp. 1P1-J18, in Japanese.

[21] J. Kohlmorgen and S. Lemm, "A dynamic hmm for on-line segmentation of sequential data," in NIPS 2001: Advances in Neural Information Processing Systems, T. G. Dietterich, S. Becker, and Z. Ghahramani, Eds., vol. 14, 2002, pp. 793-800.

[22] D. Kulić, W. Takano, and Y. Nakamura, "Incremental on-line hierarchical clustering of whole body motion patterns," in Proc. IEEE Int. Symposium on Robot and Human Interactive Communication, 2007, pp. 1016-1021.

[23] — , "Towards lifelong learning and organization of whole body motion patterns," in Proc. Int. Symposium of Robotics Research, 2007, pp. 113-124.

[24] B. Janus and Y. Nakamura, "Unsupervised probabilistic segmentation of motion data for mimesis modeling," in Proc. IEEE Int. Conf. on Advanced Robotics, 2005, pp. 411-417.

[25] L. R. Rabiner, "A tutorial on hidden markov models and selected applications in speech recognition," Proceedings of the IEEE, vol. 77, no. 2, pp. 257-286, 1989.

[26] A. K. Jain, M. N. Murty, and P. J. Flynn, "Data clustering: A review," ACM Computing Surveys, vol. 31, no. 3, pp. 264-323, 1999.

[27] D. Kulić, W. Takano, and Y. Nakamura, "Representability of human motions by factorial hidden markov models," in Proc. IEEE Int. Conf. on Intelligent Robots and Systems, 2007, pp. 2388-2393.

[28] U. Fayyad, G. G. Grinstein, and A. Wierse, Information Visualization in Data Mining and Knowledge Discovery. San Francisco, CA, USA: Morgan Kaufmann Publishers Inc., 2001.

[29] K. Yamane and Y. Nakamura, "Natural motion animation through constraining and deconstraining at will," IEEE Transactions on Visualization and Computer Graphics, vol. 9, no. 3, pp. 352-360, 2003.

[30] G. Venture, K. Ayusawa, and Y. Nakamura, "Motion capture based identification of the human body inertial parameters," in IEEE/EMBS Proc. Int. Conf. on Engineering in Medicine and Biology, 2008, pp. 4575-4578.

[31] E. R. Gansner and S. C. North, "An open graph visualization system and its applications to software engineering," Software - Practice and Experience, vol. 30, pp. 1203-1233, 1999.

[32] D. Kulić and Y. Nakamura, "Scaffolding on-line segmentation of full body human motion patterns," in Proc. IEEE/RJS Int. Conf. on Intelligent Robots and Systems, 2008, pp. 2860-2866. 